\title{
Cirurgião-dentista e técnico em prótese dentária: uma relação de mutualismo obrigatório
}

\author{
Dentist and dental prosthesis technicians: a mandatory mutualistic relationship
}

ARRUDA, Farid Jamil Silva ${ }^{1}$; LOURENÇO, Douglas ${ }^{2}$; OLIVEIRA, Marcela Duarte de ${ }^{3}$, FERNANDES, Samuel Lucas $^{4}$

1. Mestre em Prótese Dentária. Professor da Universidade Brasil, Fernandópolis, SP, Brasil.
2. Graduado em Odontologia, Universidade Brasil, Fernandópolis, SP, Brasil.
3. Graduanda em Odontologia, Universidade Brasil, Fernandópolis, SP, Brasil.
4. Mestre em Ciências Odontológicas Aplicadas. Professor da Universidade Brasil, Fernandópolis, SP, Brasil.

Endereço para correspondência:

Samuel Lucas Fernandes

Faculdade de Odontologia de Bauru - USP

Disciplina de Endodontia

Alameda Doutor Octávio Pinheiro Brisolla, 9-75

17012-901 - Bauru - São Paulo - Brasil

E-mail: samuel.lukas.usp@gmail.com

Recebido: 01.11.2017

Aceito: 12.11.2017

\section{RESUMO}

Apesar da importância decisiva do relacionamento do técnico em prótese dentária com o cirurgião-dentista, esta relação é tratada muitas vezes com descaso. $\mathrm{O}$ entrosamento entre as partes é necessário e essencial para a obtenção de um trabalho de qualidade. $\mathrm{O}$ tratamento restaurador protético integrado, centrado no paciente como foco principal do tratamento, tem transformado a prótese dentária, sendo assim, entender o conceito de time no trabalho restaurador de excelência é pensamento simples e lógico, afinal, com vistas ao sucesso do tratamento. Este artigo tem como objetivo trazer os principais fatores de congruência que são decisivos para o sucesso do tratamento em prótese dentária.

Palavras-chave: Odontólogos. Técnicos em prótese dentária. Consultórios odontológicos.

\footnotetext{
ABSTRACT

Despite the critical importance of the technical relationship with the dentist, this relationship treated with disregard. Networking is necessary and essential for quality. The integrated prosthetic restorative treatment, centered on the patient as the focus of treatment, has transformed the dental prosthesis, thus, to understand the concept of team in the restorative work of excellence is simple and logical thought, after all, with a view to the success of the treatment. This article aims to bring the main congruence of factors that are decisive for the success of dental treatment.
}

Keywords: Dentists. Dental technicians. Dental offices. 


\section{INTRODUÇÃO}

A evolução das técnicas e materiais odontológicos tem tornado reabilitações orais por meio de próteses mais acessíveis a população, com isso, substituições de dentes ausentes tem se tornado cada vez mais fácil e rápido, com objetivo de trazer resultados ao paciente com relação à estética e a função ${ }^{1-2}$.

O prognóstico de uma reabilitação protética é dependente de um correto diagnóstico e de um planejamento minucioso. A comunicação eficiente entre o técnico em prótese dentária (TPD) e o cirurgião-dentista (CD) é crítica. A colaboração mútua e íntima é fundamental para o resultado final e a longevidade do tratamento, exigindo sinergismo e disciplina entre as partes da equipe. As percepções estéticas e funcionais devem ser repassadas em detalhes ao laboratório, para que o enceramento e o planejamento possa ser executado. Transferir simultaneamente os registros funcionais, incluindo um registro preciso com o arco facial, permite ao técnico perceber o que o clínico formulou no plano de tratamento ${ }^{3}$.

O segredo do êxito do trabalho em equipe está na comunicação e o que se vê nos insucessos é a falta dela. Os lapsos no meio do processo são o "calcanhar de Aquiles" e por mais que pareçam ínfimos o resultado final perderá a qualidade a cada deslize. Portando, durante o processo clínico e laboratorial, os profissionais devem compreender suas responsabilidades, respeitando as limitações, conhecimentos individuais, assim, praticando o respeito mútuo e encorajando um ao outro, caso haja divergências em opiniões que possam interferir na qualidade final do trabalho ${ }^{4}$.

A relação de superioridade deve ser extinta, e o trabalho harmônico, e focando no resultado, baseando-se na ciência, habilidade e experiência, em detrimento da exaltação de deixando-se os títulos e graduações. A troca de informações, o repasse do conhecimento adquirido e a ponderação das experiências possibilitará ao time a satisfação com os resultados obtidos ${ }^{5}$.

Até o momento, foi pouco explorada a questão do relacionamento profissional entre os CDs e os TPDs. Portanto o objetivo deste artigo trazer as principais vias de congruência que são decisivas para o sucesso do tratamento em prótese dentária.

\section{REVISÃO DE LITERATURA}

\section{Aspectos da Comunicação entre o CD e o TPD}

A concepção de trabalhos de excelência tem relação direta com um planejamento criterioso e individualizado, tendo em vista atender às necessidades e expectativas do paciente. Esses trabalhos dependem da competência do CD e da relação com as etapas realizadas fora da boca e a ruptura desse elo levará à sua destruição.

O planejamento reabilitador é de extrema importância, pois, trabalhos de prótese dentária se tornam grandes desafios se a coleta de dados do paciente não for realizada de forma satisfatória ${ }^{6}$. É necessário, portanto, compreender as necessidades primordiais que o paciente procura para o tratamento odontológico é o principal objetivo no primeiro contato, absorvendo a maior quantidade de informações.

O diagnóstico dos problemas de comunicação não é recente. A profissão odontológica deve manter relações harmoniosas com o público, com isso, verificou o problema que existia no relacionamento entre $\mathrm{CD}$ e TPD, ressaltando a falta de interesse da classe odontológica em preparar os laboratórios de prótese dentária para que eles realizassem um melhor serviço. E até os dias atuais se observa muitas falhas na comunicação CD-TPD ${ }^{7-9}$.

Os estudos investigando ambas as partes dessa relação CD-TPD mostram que através da troca de informações e uma relação de cumplicidade podem melhorar o resultado final dos trabalhos protéticos ${ }^{5,7-8}$. Mostrando a importância tanto a etapa clínica como a laboratorial para conclusão e o sucesso do trabalho protético. Para a resolução de muitos problemas de comunicação e afinamento das partes envolvidas, pode-se lançar estratégias de ensino, desde a graduação a fim de capacitar as partes a desenvolver uma via de comunicação eficiente ${ }^{8}$.

Executar e finalizar um trabalho de boa qualidade depende da captação de informações suficientes, que são adquiridas através de: modelo antagonista, moldagem para modelo mestre, registros de articulação, modelo dos provisórios, protocolo de fotografia e prescrição do trabalho, devendo ser compartilhadas entre $\mathrm{CD}$ e TPD ${ }^{10-11}$. Atualmente novas tecnologias como o uso de escaneamento intraoral e tecnologia $\mathrm{CAD} / \mathrm{CAM}$ tem se incluído a importância do aprendizado dessas novas tecnologias e o afinamento dos profissionais para um melhor uso dessas tecnologias ${ }^{12}$.

A comunicação eficiente é o segredo do sucesso do trabalho em equipe. No caso da prótese dentária, a qualidade final do trabalho protético está diretamente relacionada à fase clínica, de responsabilidade do $\mathrm{CD}$, e a fase laboratorial, desenvolvida pelo TPD. Com isso, a comunicação existente entre esses dois profissionais deverá ser estreita e harmônica, para que se obtenha sucesso no trabalho final ${ }^{13}$.

Os principais pontos a serem observados são:

Modelo de Estudo

A obtenção de modelos de estudo da arcada superior e inferior permitem uma visualização tridimensional dos dentes e tecidos adjacentes, impossível de se obter clinicamente. Através dos modelos podem-se observar 
detalhes gengivais, inclinações, formas dentais e relação dos dentes em conjunto e com seus antagonistas ${ }^{14}$.

\section{Comunicação da Cor}

Um dos requisitos mais importantes para o desenvolvimento da harmonia, estética e do sucesso final das restaurações, é a comunicação de cor, pois, torna a realização das reconstruções diretas e indiretas um processo complexo devido à natureza tridimensional da $\mathrm{cor}^{15}$.

A cor é um dos principais determinantes estéticos de restaurações diretas e indiretas. Habitualmente, a seleção de cor é realizada por método de comparação visual por meio de escalas convencionais que, frequentemente, resulta na determinação imperfeita das cores, devido às deficiências técnicas das escalas e a fatores subjetivos inerentes às técnicas e ao observador ${ }^{16}$.

Escalas de cores são instrumentos são os instrumentos mais utilizados para a escolha de cor e para a comunicação codificada entre $\mathrm{CD}$ e $\mathrm{TPD}^{17}$. Na Odontologia contemporânea a concordância entre a cor do elemento dentário e dos materiais restauradores ainda é fundamental ${ }^{17}$ e as escalas têm suas limitações em relação ao fim a que se presta, o principal motivo, provavelmente, é a grande variação de nuances de cor que os elementos dentários apresentam $^{10}$. A seleção da cor na Odontologia é um desafio para a prática clínica e o $\mathrm{CD}$ tem a necessidade de estudar a ciência das cores. Atualmente com o emprego de aparelhos para a seleção de cor através do método digital permite a minimização das possíveis falhas relativas e esta etapa do procedimento. Atualmente, existem no mercado alguns aparelhos com esta finalidade tais como colorímetros e espectrofotômetros ${ }^{10}$.

A seleção de cor de um ou mais elementos dentais deve abranger uma sintonia muito grande entre CD e TPD, sendo um procedimento relativamente complexo e realizado praticamente com o uso de uma escala de cores e um mapeamento desenhado em papel pelo CD ou TPD, tendo a fotografia digital como um auxiliar nesta comunicação ${ }^{10}$. (ASA)

Montagem de Modelos em Articulador Semi Ajustável

No arsenal de instrumentos que o $\mathrm{CD}$ dispõe para executar o seu trabalho, o ASA tornou-se peça fundamental e indispensável nas reconstruções tanto simples como complexas. O uso rotineiro do ASA tornou-o o instrumento mais utilizado dentre outros usados em reabilitação bucal, entretanto, a sua utilização envolve conhecimento específico das qualidades e limitações desses instrumentos ${ }^{18}$.

A montagem incorreta dos modelos de gesso em ASAé um dos erros mais comuns em reabilitação oral, portanto, devemos dar atenção especial nessa importante etapa19. A precisão da montagem em ASA tem implicações significativas em toda a etapa de diagnóstico e planejamento, e posteriormente na execução laboratorial.
Interferindo diretamente na adaptação dos trabalhos na boca e interfere no número de tipos de ajustes clínicos ${ }^{19}$.

\section{Fotografia Digital Odontológica}

A Odontologia deu um grande salto nos últimos dez anos em documentação e arquivamento de dados devido à revolução da informática e suas consequências desde a compilação de dados descrevendo condutas diárias em prontuários, até o gerenciamento de imagens e vídeos de condutas e procedimentos ${ }^{20}$.

A fotografia permite ao CD perpetuar o momento clínico em que se depara, e é de extrema importância para a comunicação uma vez que expressa de maneira ímpar a condição em que o paciente se encontra e pode levar os trabalhos a níveis elevados de qualidade e principalmente propicia a individualização do caso, o planejamento e a visualização prévia por meio de ensaios digitais. Atualmente a fotografia faz parte do arsenal de um odontólogo que prima a qualidade de seus serviços ${ }^{21}$.

Os laboratórios de prótese dentária utilizam dessa tecnologia como documento cadastral, acompanhamento de casos clínicos, divulgação de trabalhos realizados e principalmente para realizarem um trabalho protético mais fiel, com o objetivo de se aproximar da cor, formato, textura e das demais características do dente natural. Assim, os TPDs utilizam desses artifícios para uma visualização clínica imediata, impensável pouco tempo atrás, quando era necessário o processo de revelação de filmes e possuíam apenas modelos de gesso, escala de cor e desenhos fornecidos pelos $\mathrm{Cds}^{21}$.

Em uma Odontologia de alto nível, o diferencial entre os grandes profissionais não é mais a qualidade do trabalho, já que este se tornou inerente ao profissional e é fundamental. $\mathrm{O}$ que pode diferenciar um profissional de outro é a forma de divulgação e apresentação do seu trabalho; pois é através da fotografia que o trabalho poderá ser ainda mais valorizado ${ }^{21}$.

A utilização de ferramentas digitais proporciona uma interação maior entre a equipe, além de ser uma ferramenta excelente para a comunicação com o paciente. Atualmente a comunicação visual é de fundamental importância para o sucesso, possibilitando antever passos clínicos, laboratoriais e até mesmo o resultado esperado para o caso $^{22}$.

Enceramento Diagnóstico

Esta é uma etapa que extrapola a fase de planejamento, podendo ser um demonstrativo do trabalho a ser executado, indispensável para as disposições, formas e estética correta dos dentes, possibilitando a visualização de uma perfeita harmonia nos movimentos mandibulares e morfologia oclusal, como também um prognóstico funcional e estético $^{23-25}$.

$\mathrm{Na}$ maior parte dos trabalhos, o enceramento diagnóstico é realizado pelo TPD, portanto, o CD molda a boca do paciente, procede o vazamento que resulta no 
modelo de gesso da boca deste paciente e executa a montagem em articulador. No enceramento diagnóstico, os dentes são esculpidos um a um, em processo quase artesanal, exigindo uma série de adaptações, consistindo em um jogo de ajustes para ocupar os espaços edêntulos e com os movimentos mandibulares ${ }^{26}$.

O enceramento diagnóstico é indiscutível para planejamento de reabilitações protéticas ${ }^{25-26}$, a prática desta técnica vem sendo de uso quase que exclusivo de TPDs e CDs habilitados e mais experientes, sempre requerendo um entrosamento bastante amplo destes profissionais ${ }^{26}$. A disseminação do conhecimento e emprego dessa técnica é de fundamental importância para o desenvolvimento de uma Odontologia de excelência.

Em dentes anteriores a confecção de facetas e/ou fragmentos cerâmicos caracteriza-se por ser um procedimento primeiramente estético e capaz de proporcionar a preservação de estrutura dental sadia, se comparado ao preparo de coroas. $\mathrm{O}$ enceramento e o planejamento propiciam uma Odontologia minimamente invasiva $^{27}$. A partir desse ensaio no modelo, pode-se transferir para a boca por meio da confecção de um mockup, que tem por finalidade dar resultado imediato no planejamento do trabalho a ser executado10, além de menor risco biológico, estético e funcional, demonstração de várias opções de tratamento, simulação do resultado estético e a aceitação prévia do tratamento pelo paciente ${ }^{28}$.

\section{Planejamento}

O planejamento correto passo pela compreensão das necessidades primordiais de um paciente que procura tratamento odontológico, tendo como principal objetivo identificar a expectativa e o grau de exigência com relação ao tratamento a ser realizado. Esse delineamento será feito à luz dos referenciais estéticos que o paciente almeja e não em função do profissional ou dos materiais de sua preferência.

Nos dias atuais, há uma grande demanda pela busca da beleza, pelo fato das pessoas estarem insatisfeitas com a colocação, formato e disposição dos dentes, não se encaixando nos estereótipos ditados pela sociedade moderna, resultando em baixa autoestima ${ }^{29}$. É indispensável que o $\mathrm{CD}$ esteja atento às exigências e desejos dos pacientes para que possa atendê-los integralmente, devolvendo não apenas a estética, mas também a saúde através do seu bem-estar psíquico e social $^{16}$

O planejamento deve visar ainda a conservação da estrutura dentária, visto que uma vez perdida essa estrutura não poderá se regenerar. Usualmente o $\mathrm{CD}$ realiza o preparo dentário, para posteriormente reestabelecer a estética e função por meio de uma peça preparada pelo TPD. Essa prática gera muitas vezes desgastes desnecessários e a realização de procedimentos de baixa previsibilidade. Portanto o planejamento deve ser baseado em estudos prévios e em um diagnóstico bem estabelecido.

A Odontologia contemporânea se vale de métodos auxiliares de diagnóstico e ferramentas de planejamento que minimiza os erros e possibilitam antever muitas situações clínicas. Fotografias, enceramentos diagnósticos e planejamentos digitais são ferramentas indispensáveis para uma avaliação detalhada de todos os fatores que interferem na harmonia e simetria dos elementos que compõem o sorriso, possibilitando assim, bons resultados30. As possibilidades devem ser discutidas inicialmente com o paciente pelo $\mathrm{CD}$ e posteriormente o CD com o TPD, a fim de criar uma cadeia interativa que permita a entrega de um trabalho de qualidade.

\section{CONCLUSÃO}

A captação de informações suficientes do paciente como: modelos de estudo, tomada de cor, montagem em asa, fotografias digitais, associado a uma relação de mutualismo com o laboratório, permite realizar um planejamento adequado visando a estética e função, e alcançar a qualidade final desejada para o trabalho.

A filosofia do tratamento restaurador protético integrado, centrado no paciente como foco principal do tratamento, tem transformado a prótese dentária, sendo assim, entender o conceito de time no trabalho restaurador de excelência é pensamento simples e lógico, afinal, com vistas ao sucesso, CD e TPD associados numa ação comum, em conectividade e cooperação, terão muito mais previsibilidade e conforto nas decisões, estratégias e execução. A nova Odontologia restauradora protética de parceria, de profissionalismo e amizade, abre os canais para a comunicação, para a divisão de responsabilidades e, especialmente para o bem-estar do paciente.

\section{REFERÊNCIAS}

1. Castro JCM, Castro MAM, Pedrini D, Panzarini SR, Pelielo AR. Prótese adesiva: uma opção estética, conservadora e funcional. RGO. 2006;54(3):225-9.

2. Pineli 1AP, Marra j, Fais 1MG, Silva RHBT, Guaglianoni DG. Análise da condição de higiene oral de pacientes usuários de prótese parcial fixa. ROBRAC. 2007;16(42):1-6.

3. Fradeani M, Barducci G. Comunicação com o laboratório enceramento diagnóstico. In: Tratamento protético - uma abordagem sistemática à integração. 2 ed. Curitiba: Quintessence; 2009.

4. Drago CJ. Clinical and laboratory parameters in fixed prosthodontic treatment. La Crosse: Gundersen Clinic; 1996. p. 233-8.

5. Gouvêa CVD, Faria MA, Paula LD. Avaliação da relação profissional entre o cirurgião dentista e o técnico em prótese dental, 
interferindo na qualidade final da prótese. Odontologia Clin.-Cient. 2006;5(3):217-23

6. Marcondes R, Calgaro M. O desafio estético com próteses anteriores unitárias: o planejamento com integração clínico-laboratorial. Rev Dent Press. 2008;5(4):31-47.

7. Stewart CA. An audit of dental prescriptions between clinics and dental laboratories. Br Dent J. 2011;211(3):E5.

8. Sui L, Wu X, Wu S, Gao P, Li R. The quality of written instructions for dental prostheses in China. J Prosthodont. 2014;23(8):602-9.

9. Parry GR, Evans JL, Cameron A. Communicating prosthetic prescriptions from dental students to the dental laboratory: is the message getting through? J Dent Educ. 2014;78(12):1636-42.

10. Kina S, Bruguera A. Invisível - restaurações estéticas cerâmicas. Maringá: Dental Press; 2007.

11. Feuerstein P. New changes in CAD/CAM: part 1.lnside dentistry. North Billerica; 2007.

12. Machado RMP, Almeida JRV, Martins F, Ribeiro CF, Tirzah HK. Condição de envio dos modelos de trabalho e comunicação entre cirurgiões dentistas e técnicos em prótese dentária do município de Aracaju - Sergipe na confecção e prótese fixa metalocerâmica. Odontologia Clin.-Cient. 2010;9(3):257-62.

13. Phoenix RD, Cagna DR, Defreest C. Prótese parcial removível: clínica de Stewart. 3 ed. Chicago: Quintessence; 2007.

14. Volpato CAM, Baratieri LN, Monteiro Júnior S. Análise instrumental da cor em odontologia: considerações básicas. Rev Dent Press Estet. $2005 ; 2(1): 21-31$.

15. Rosa V, Bona AD. Seleção de cor em consultórios: das escalas convencionais ao espectrofotometro. Clin Int J Braz Dent. 2007;3(1):62-8.

16. Alves GN, Aras WMF. Percepção de pacientes em relação à estética dentária. Rev.Saude.Com. 2014;10(2):161-71.

17. Smith GP. The responsability of the dentist toward laboratory procedures in fixed and removable partial denture prosthesis. J Prosthet Dent. 1963;13(2):295-301.

18. Bini N, Carvalho WR, Bruno MAD, Gouvêa CVD. Articuladores e seu uso na odontologia. Odontol Clin Cient. 2011;517-21.

19. Zanetti AL, Ribas R. Técnica de transferência direta do molde da arcada superior do paciente para o articulador semi-ajustável. Rev Bras Prot Clin Lab. 2001;3(15):372-81.

20. Oliveira JP. Fotografia e vídeo digital: a nova fronteira da odontologia. Rev Dent Press Est. 2005;2(1):117-32.

21. Faccirolli IYO, Paula RF. O mundo da fotografia. In: Faccirolli IYO. A arte da fotografia digital na odontologia. São Paulo: Ed. Santos; 2010.p. 5-12.

22. Coachman C, Calamita M. Digital smile design: A tool for treatment planning and communincation in esthetic dentistry. Quintessence Dent Technol 2012;35:103-12

23. Bassanta AD. A importância do enceramento de diagnóstico na reabilitação oral. Rev Bras Odontol. 1992;49(6):10-15.

24. Machado MAC. Enceramento de diagnóstico para um contorno anatômico. Rev Paul Odontol. 1994;16(3):18-30.
25. Shirata OC. As influências dos determinantes da oclusão no enceramento de diagnóstico. Rev Odontol. 1999;7(16):46-50.

26. Pompeu JGF, Prado VLG. Técnica fácil e rápida de enceramento diagnóstico utilizada no atendimento odontológico público na Universidade Federal do Piauí - UFPI. Int J Dent. 2004;3(1):308-11.

27. Souza SM, Marson FC, Sensi LG, Brandenburgo G, Araújo E. Preparo de dentes para faceta de porcelana guiado por ensaio diagnóstico. Clin Int J Braz Dent. 2006;2(4):392-401.

28. Silva TB, Lopes LV, Oliveira MBRG, Takano ERA, Cardoso PC. O uso do mock-up no planejamento de restaurações cerâmicas. Rev Assoc Paul Cir Dent. 2010,64(1):43-9.

29. Sakamoto Júnior AS, Yuen MSY, Higashi, Liu J, Hirata R, Gomes JC. Protocolo clínico para laminados cerâmicos. J ILAPEO. 2002;6(1):15-9.

30. Shibasaki DN, Martins VL, Leal CL, Queroz APV, Mathias P, Cavalcanti AN. Recursos contemporâneos do planejamento estético integrado. Rev Bahiana Odontol. 2013;4(2):147-57. 